$18 \%$ of these patients had positive surgical margins without extraprostatic extension (defined as capsule incision into tumor). Capsule incision had an independent negative prognostic impact in all groups analyzed.

This information is important to all surgeons considering nerve-sparing radical prostatectomy procedures.

\author{
Dr. Andreas Bohle \\ Professor of Urology \\ HELIOS Agnes Karll Hospital \\ Bad Schwartau, Germany \\ E-mail: boehle@urologie-bad-schwartau.de
}

\title{
Combination of adjuvant hormonal and radiation therapy significantly prolongs survival of patients with pT2-4 pN+ prostate cancer: results of a matched analysis \\ Briganti A, Karnes RJ, Da Pozzo LF, Cozzarini C, Capitanio U, Gallina A, Suardi N, Bianchi M, Tutolo M, Salonia A, Di Muzio N, Rigatti P, Montorsi F, Blute M \\ Department of Urology, Vita-Salute University, San Raffaele Scientific Institute, Milan, Italy \\ Eur Urol. 2011; 59: 832-40
}

Background: Previous prospective randomised trials have shown a positive impact of adjuvant radiation therapy (RT) in patients with locally advanced prostate cancer. However, none of these trials included patients with lymph node invasion (LNI).

Objective: The aim of this study was to assess the impact of combination adjuvant hormonal therapy (HT) and RT on the survival of patients with prostate cancer and histologically documented lymph node metastases (pN+). Design, Setting, and Participants: Data on 703 consecutive patients with LNI treated with radical prostatectomy, pelvic lymph node dissection, and adjuvant treatments between September 1986 and November 2002 at two large academic institutions were reviewed.

Measurements: For study purposes, patients treated with adjuvant HT plus RT and patients treated with adjuvant HT alone were matched for age at surgery, pathologic T stage and Gleason score, number of nodes removed, surgical margin status, and length of follow-up. Differences in cancer-specific survival (CSS) and overall survival (OS) were compared using the Kaplan-Meier method and life table analyses.

Results and Limitations: Following the matching process, 117 pT2-4 pN1 patients of $171(68.4 \%)$ treated with adjuvant HT plus RT (group 1) were compared with 247 pT2-4 pN1 patients of 532 (46.4\%) receiving adjuvant HT alone (group 2). After matching, the two groups of patients were comparable in terms of pre- and postoperative characteristics (all $\mathrm{p} \geq 0.07$ ). Mean follow-up was 100.8 mo (median: $95.1 \mathrm{mo}$; range: 3.5-229.3 mo). Overall, prostate CSS and OS rates at 5, 8 , and $10 \mathrm{yr}$ were $90 \%, 82 \%$, and $75 \%$, and $85 \%, 70 \%$, and $60 \%$, respectively. Patients treated with adjuvant RT plus HT had significantly higher CSS and OS rates compared with patients treated with HT alone at 5, 8, and $10 \mathrm{yr}$ after surgery $(95 \%, 91 \%$, and $86 \% \mathrm{vs} 88 \%, 78 \%$, and $70 \%$, and $90 \%, 84 \%$, and $74 \%$ vs $82 \%, 65 \%$, and 55\%, respectively; $p=0.004$ and $p<0.001$, respectively). Similarly, higher survival rates associated with the combination of HT plus RT were found when patients were stratified according to the extent of nodal invasion (namely, two or fewer vs more than two positive nodes; all $\mathrm{p}$ $\leq 0.006$ ). Lack of standardised HT and RT protocols represents the main limitations of our retrospective study. Conclusions: Adjuvant RT plus HT significantly improved CSS and OS of pT2-4 pN1 patients, regardless of the extent of nodal invasion. These results reinforce the need for a multimodal approach in the treatment of node-positive prostate cancer. 


\title{
Editorial Comment
}

Patients with positive lymph nodes after radical prostatectomy should receive hormonal therapy, this is common urological knowledge. Should patients with positive lymph nodes also receive adjuvant radiotherapy? This question was addressed in a large retrospective matched-pair study. $76 \%$ of $\mathrm{N}+$ patients received adjuvant hormonal therapy alone and $24 \%$ received both adjuvant hormonal therapy and radiotherapy.

Interestingly, and of large clinical importance, the authors found survival advantages in favour of adjuvant radiotherapy in all subgroups analyzed. The results of this study should give cause for an appropriate prospective trial and may result in a shift of current clinical recommendations.

\author{
Dr. Andreas Bohle \\ Professor of Urology \\ HELIOS Agnes Karll Hospital \\ Bad Schwartau, Germany \\ E-mail:boehle@urologie-bad-schwartau.de
}

NEUROLOGY \& FEMALE UROLOGY

Correlations between pretransplant dialysis duration, bladder capacity, and prevalence of vesicoureteral reflux to the graft

Inoue T, Satoh S, Saito M, Numakura K, Tsuruta H, Obara T, Narita S, Horikawa Y, Tsuchiya N, Habuchi T 1 Department of Urology, Akita University School of Medicine, Akita, Japan. 2 Division of Renal Replacement Therapeutic Science, Akita University School of Medicine, Akita, Japan

Transplantation. 2011; 92: 311-5

Background: Urinary bladder capacity is reduced in patients undergoing long-term dialysis, which may increase the risk of vesicoureteral reflux (VUR) to a transplanted kidney. This study investigated the correlations between dialysis duration, pretransplant and posttransplant bladder capacity, and prevalence of VUR to the graft.

Methods: Voiding cystography was performed in 101 adult renal transplant recipients without neurogenic disorders immediately before and 1 year after transplantation to evaluate bladder capacity and VUR. Nonstented extravesical antireflux ureteroneocystostomy was performed in all patients.

Results: The median dialysis duration and pretransplant bladder capacity were 32 months (range 1-426 months) and $120 \mathrm{~mL}$ (range 15-450 mL), and 21 patients (20.8\%) underwent dialysis for more than 120 months, and 30 patients $(29.7 \%)$ had a pretransplant bladder capacity of less than $80 \mathrm{~mL}$. Dialysis duration was correlated with pretransplant bladder capacity $(\mathrm{R}=0.466, \mathrm{P}<0.001)$. Bladder capacity expanded more than 6 -fold from pretransplantation to posttransplantation, and all recipients had a bladder capacity greater than $150 \mathrm{~mL}$ at 1 year posttransplantation. Thirty patients had VUR to the graft. Dialysis duration longer than 60 months $(\mathrm{P}=$ $0.021)$ and pretransplant bladder capacity of less than $130 \mathrm{~mL}(\mathrm{P}=0.024)$ were associated with VUR. VUR was associated with lower graft function.

Conclusions: Although bladder capacity decreased because of long-term dialysis, it exceeded $150 \mathrm{~mL}$ at 1 year posttransplantation. A small bladder can be used in renal transplantation, but it may increase the risk of VUR. 